\title{
MODELOS PEDAGÓGICOS NA ESCOLA RURAL PARA VIVER A DEMOCRACIA.
}

\author{
Roser Boix Tomàs \\ Facultad Educación, Universidad de Barcelona. \\ https://orcid.org/0000-0001-9000-7967 \\ Josep Rius Roig \\ Escuela Rural Virolai, Barcelona. \\ https://orcid.org/0000-0002-4470-7134
}

\section{RESUMO}

Atualmente, muitas escolas estão implementando modelos pedagógicos que apostam na democracia escolar. Estes são modelos em que as relações entre as pessoas da sala de aula e na escola não seguem uma hierarquia vertical clássica, mas a partir de uma abordagem horizontal e onde a organização tradicional do currículo por assunto se rompe para utilizar os conteúdos e as competências de forma mais transversal; a diversidade torna-se um valor pedagógico em vez de um problema. O objetivo principal do artigo é aprofundar o conceito de democracia escolar na escola rural como um processo que é necessário para viver, como já disse Dewey em 1916, em seu famoso livro Democracia e Educação: Uma Introdução à Filosofia da Educação; e isso pode ser feito a partir de modelos pedagógicos onde o trabalho colaborativo e as estratégias ativo participativas favorecem a vivencia dos valores plurais, humanos e inclusivos na escola e ademais respondem a abordagens de justiça social, igualdade, equidade e participação, compartilhando-as com a comunidade de educação rural em todos os aspectos relevantes do centro e o agrupamento de escolas rurais, tanto do ponto de vista da gestão quanto dos aspectos relacionados a ela com a aprendizagem dos alunos.

Palavras chave: escola rural, democracia, valores plurais, aula multiseriada, modelos pedagógicos

\section{MODELOS PEDAGÓGICOS EN LA ESCUELA RURAL PARA VIVIR LA DEMOCRACIA.}

\section{RESUMEN}

En la actualidad, muchas escuelas están implantando modelos pedagógicos que apuestan por una democracia escolar. Se trata de modelos en los que las relaciones entre las personas del aula y de la escuela no siguen una jerarquía clásica vertical sino que parten de un planteamiento horizontal y en donde la organización tradicional del currículum por materias se rompe para utilizar formas de organizar los contenidos y las competencias de forma más transversal; la diversidad se convierte en un valor pedagógico en lugar de un problema. El artículo tiene como objetivo principal profundizar en el concepto de democracia escolar en la escuela rural como un proceso que es necesario vivirlo, como ya nos decía Dewey en el año 1916, en su famoso libro Democracy and Education: An Introduction to the Philosophy of Education; y ello puede llevarse a cabo a partir de modelos pedagógicos donde el trabajo colaborativo y las estrategias didácticas activo-participativas favorecen la vivencia de los valores plurales, humanos e inclusivos en la escuela; pero además, responden a planteamientos de justícia social, igualdad, equidad y participación, compartiéndolos con la comunidad educativa rural en todos los aspectos relevantes del centro y de la agrupación de escuelas rurales, tanto desde la perspectiva de la gestión como de los aspectos propiamente relacionados con los aprendizajes de los alumnos.

Palabras clave: escuela rural, democracia, valores plurales, aula multigrado, modelos pedagógicos

\section{PEDAGOGICAL MODELS IN THE RURAL SCHOOL TO LIVE DEMOCRACY.}

\section{ABSTRACT}

At present, many schools are implementing pedagogical models that bet on a school democracy. These are models in which the relationships between the people in the classroom and the school do 
not follow a classic vertical hierarchy, but start from a horizontal approach and where the traditional organization of the curriculum by subject is broken to use ways to organize the contents and competences more transversally; diversity becomes a pedagogical value instead of a problem. The main objective of the article is to deepen in the concept of school democracy in the rural school as a process that is necessary to live it, as Dewey told us in 1916, in his famous book Democracy and Education: An Introduction to the Philosophy of Education; and this can be carried out from pedagogical models where collaborative work and active-participative didactic strategies favor the living of plural, human and inclusive values in the school; but also respond to approaches of social justice, equality, equity and participation, sharing them with the rural education community in all relevant aspects of the center and the grouping of rural schools, both from the perspective of management and the aspects related to it with the learning of the students.

\section{INTRODUCCIÓN}

"La enseñanza debe ser por la acción. La educación es la vida; la escuela es la sociedad" (Dewey, 1916)

La escuela es un reflejo de la sociedad, pero no se debe ver como espejo de la sociedad presente si no el de la potencialidad futura. Que en las escuelas se trabaje la democracia, entendida no sólo como un sistema político, sino como una forma de vida basada en valores universales y en derechos humanos, donde los individuos se sientan libres, respetados, escuchados, participantes en la toma de decisiones y responsables de las mismas es indispensable para avanzar hacía una mejora social.

Desde la perspectiva de la escuela rural, el reflejo es más cercano a la realidad ya que se parte de un aula multigrado, donde se encuentran niños y niñas de edades, intereses y capacidades distintas, como pasa en la sociedad. Además suelen ser comunidades más reducidas en número de miembros, hecho que propicia la flexibilidad en la gestión del grupo y permite desarrollar los valores democráticos desde una pluralidad más cercana. En estos casos, los maestros no pueden ser meros transmisores de contenidos, la verticalidad desaparece para dar lugar a la horizontalidad en la que el alumnado se convierte en miembro activo de la organización escolar, participando directamente en el proceso de aprendizaje propio y de los demás. Es esta participación la que convierte los niños en ciudadanos críticos e implicados, puesto que desarrollan herramientas como el diálogo que les permite argumentar y cuestionar ideas, una actitud imprescindible para seguir evolucionando como sociedad democrática.

Este artículo pretende descubrir algunas de las claves de la escuela democrática destinadas a comunidades rurales donde los centros escolares forman una parte muy relevante de su vida social. Una primera parte está dedicada a la democratización 
obligatoria de la escuela rural por su propia idiosincrasia y las dinámicas que de forma natural la han llevado a este proceso. Por otra parte, se detallan los modelos pedagógicos y las actividades concretas que procuran, desde un punto de vista práctico, ayudar a los maestros a tender hacía el uso de unas metodologías mixtas y activas, en las que el libro de texto no sea el centro y la guía de las aulas sino un recurso más; donde el empoderamiento del alumnado participativo y responsable sea el motor de una mejor sociedad democrática futura.

\section{LA ESCUELA RURAL COMO ESCUELA DEMOCRÁTICA}

Alain Touraine (2011, p16) apela a la necesidad de reconstruir la vida social, "y en poner fin a la dominación de la economía sobre la sociedad, lo que exige el recurso a un principio cada vez más general e incluso universal que se puede llamar de nuevo los derechos del hombre (o mejor humanos) que debe engendrar formas nuevas de organización, de educación y de gobernanza" para ser capaces, entre otras cosas, de exigir un respeto más real a la dignidad de todos los seres humanos. La dignidad humana es uno de los principios fundamentales de la democracia, junto con la libertad y los derechos de todos y cada uno de los miembros de la colectividad y la educación el medio a través del cual fomentamos (o debemos fomentar) esa dignidad; la democracia no es solo una forma de gobierno sino un modo de vivir, una forma de vida como nos decía Freire (1974), en la que no existen barreras por razón clase, sexo, raza y territorio nacional, y en la realización de una forma de vida social que se sustenta en los intereses que penetran recíprocamente y en donde el progreso o reajuste merece una importante consideración, en realidad, hace a una sociedad democrática más interesada que otras en organizar una educación deliberada y sistemática, Dewey (1995).

\section{PRÁCTICA PEDAGÓgICA MULTIGRAdO Y VALORES PLURALES DEMOCRÁTICOS}

Los valores plurales democráticos que caracterizan la democracia pretenden destacar las cualidades de las sociedades y de los propios individuos que la conforman; son fundamentales para la sociedad actual porque forman la base de la misma y de su funcionamiento diario. Garantizan, además, uno de los derechos humanos básicos como es el derecho a una educación de calidad y equidad para toda la población. 
Entre los valores de la democracia distinguimos la libertad, la justicia, la igualdad, la participación, la solidaridad, el diálogo y la tolerancia. Valores, que entre otros, impregnan la práctica pedagógica del aula multigrado, siempre desde la perspectiva de una clara limitación al autoritarismo, en donde se impone el poder de una figura de autoridad unitaria que suele ser el maestro y de un sistema educativo que como nos señala Fromm (2005) en su libro El miedo a la libertad, se halla determinado por la eliminación de la libertad y de los sentimientos del niño, para imponerle otros ajenos por medio de métodos reprochables.

Como decíamos, la práctica pedagógica del aula multigrado con una intencionada limitación al autoritarismo se sustenta bajos unos fundamentales valores democráticos, de entre ellos vamos a destacar los siguientes:

- Libertad. El planteamiento activo-participativo en el aula multigrado conlleva que el niño aprende a gestionar su tiempo para poder realizar las tareas trabajando activamente y en el marco de un contexto donde se fomenta la autonomía de aprendizaje, tal y como ya nos señalaba Piaget (1949) "no se aprende a experimentar simplemente viendo experimentar al maestro o dedicándose a ejercicios ya totalmente organizados: sólo se aprende a experimentar probando uno mismo, trabajando activamente, es decir, en libertad y disponiendo de todo su tiempo" (p. 39); compartir espacio y tiempo con otros niños de edades distintas implica la necesidad de aprender a auto-gestionar el tiempo, de tomar decisiones en relación a los compromisos adquiridos en un contexto de libertad sin embargo, no debe confundirse este concepto abierto de libertad en el desarrollo de la práctica pedagógica como un estado de aislamiento individual sino como una capacidad que permite de forma colectiva, que el grupo clase pueda alcanzar los objetivos comunes traduciéndolos en acciones y superando los problemas colectivos. "Renunciar a la libertad es renunciar a ser hombre” nos decía Rousseau (2017).

- Justícia. En el aula multigrado de la escuela rural se fomenta el reconocimiento y la participación de todos los miembros de la clase, inter-edades e inter-niveles, favoreciendo acciones y tareas concretas para que aprendan quienes son y de donde vienen, cual es la cultura y el entorno rural en el que se encuentran inmersos, el papel de la escuela y de la comunidad educativa en la dimensión territorial. La diversidad natural existente en el aula crea un clima de respeto hacia las personas, autoestima y 
autoconocimiento. El alumnado comparte con sus compañeros sus conocimientos sobre su propio contexto cultural y ambiental.

- Participación. La participación es un derecho regulado desde 1948 en la Declaración de los Derechos Humanos, artículo 19 en el que se dice que: "Todo individuo tiene derecho a la libertad de opinión y de expresión; este derecho incluye el de no ser molestado a causa de sus opiniones, el de investigar y recibir informaciones y opiniones, y el de difundirlas, sin limitación de fronteras, por cualquier medio de expresión"; es además, el ejercicio democrático de la igualdad de oportunidades en la toma de decisiones y en el desarrollo de tareas. En el aula multigrado los alumnos participan activamente como sujetos involucrados, en la consecución de objetivos comunes. A través de metodologías didáctica activo-participativas se fomenta esta participación no solo en la realización de actividades, sino en su diseño, en la toma de decisiones sobre su pertinencia, se cuestionan los temas a tratar, se argumentan las resoluciones y se centran en los intereses y necesidades de aprendizaje reales de los niños. La participación adquiere un sentido porque permite la construcción de un proyecto común donde se pueden lograr aprendizajes personales y colectivos. Todo ello fortalece las relaciones afectivas, de amistad, y el sentimiento de pertenencia a la comunidad educativa y a la rural propiamente.

Los maestros rurales son, sin duda alguna, los principales gestores de la participación de los alumnos en los procesos de aprendizaje. Se promueve una participación sentida y real que surge de forma natural entre los niños de diferentes edades, más allá de la verticalidad; el modelo pedagógico activo-participativo del aula multigrado facilita que el maestro diseñe y ofrezca espacios de participación entre los mismos alumnos y entre los alumnos, el maestro y la comunidad educativa.

- Solidaridad y tolerancia. En la escuela rural, por la convivencia de distintas edades se fomenta la solidaridad y la tolerancia; los pequeños aprenden de los mayores y los mayores aprenden a aprender cuando enseñan a los pequeños, incluso aprenden de ellos. Escuchar y respetar las opiniones y argumentos de los compañeros de diversas edades, tolerar el turno de palabra, considerar el ritmo de aprendizaje de los compañeros, ser 
empático con los problemas de los demás indiferentemente de las edades son elementos clave para la construcción y afianzamiento de los principios de solidaridad y tolerancia.

- Diálogo. El diálogo es un pilar fundamental del aprendizaje y de la convivencia dentro de la escuela y de la sociedad, en general; la Real Academia Española define el diálogo como Plática entre dos o más personas, que alternativamente manifiestan sus ideas o afectos; de este modo, el diálogo enriquece a los interlocutores, permite la participación de todos los alumnos y favorece las relaciones inter e intrapersonales,; la construcción de significados compartidos dentro del aula multigrado facilita la comunicación bidireccional entre los alumnos y entre los alumnos y el maestro; se aprende de los otros y con los otros, se ejercita en el método de la escucha y argumentación para poder aplicarlo también fuera de la escuela; y especialmente se aprende a ser paciente, a respetar los tiempos de los otros y a reflexionar sobre las aportaciones de los otros.

- Inclusión e igualdad. La escuela rural promueve las culturas y las prácticas inclusivas dentro del aula multigrado debido básicamente a la proximidad y al conocimiento profundo que cada maestro tiene del alumno; ello permite valorar igualitariamente a todo el alumnado huyendo de la homogeneización del grupo-clase y focalizando al niño como único dentro de la propia escuela. La ayuda entre los propios compañeros contribuye, a generar aprendizajes colaborativos, la enseñanza en equipo, resultando que el proceso de enseñanza-aprendizaje sea menos competitivo y angustiante para ellos. Si, además, las programaciones curriculares son diseñadas de forma que la diversidad del aula multigrado sea utilizado como un recurso didáctico y fomentando la participación activa de los alumnos, se promueve la inclusión de forma natural, y la auto-regulación del propio proceso de aprendizaje. De todas formas, como nos dice Escudero (2012) la verdadera inclusión educativa requiere y depende, a su vez, de una perspectiva de mayor alcance como la inclusión social.

Las práctica pedagógicas multigrado, desde la perspectiva de una clara limitación al autoritarismo, permiten la realización de actividades vinculadas a los intereses y necesidades de los alumnos, pero deben ser intencionadas, estimuladas y sensibles a las predilecciones de sus protagonistas; y en ningún momento improvisadas porque puede 
quedar reducido a una mera distribución de tareas, en las que no se de una igualdad de oportunidades real en su desarrollo y sistematización.

\section{VIVIR Y HACER VIVIR LA DEMOCRACIA EN LA ESCUELA RURAL}

Los valores plurales que fundamentan la escuela rural desde la perspectiva de una clara limitación al autoritarismo, deben ser vividos dentro del aula por parte de los alumnos, los maestros e incluso la propia comunidad rural. Un ambiente educativo marcado por estos principios es un ambiente que permite vivir la democracia como una realidad no acabada, sino como un proyecto personal y colectivo que se va construyendo. El desarrollo de la convivencia democrática en el aula implica que los componentes del proceso de enseñanza-aprendizaje y sus prácticas pedagógicas contemplen en sí mismos valores plurales democráticos. La formación del maestro también debe contemplar la instrucción como una comunicación multidireccional ofreciendo reflexiones, propuestas e instrumentos didácticos que les permitan poner en práctica la dialéctica, deliberada y participativa de todos los miembros de la comunidad educativa en la elaboración de los proyectos comunes de centro, y en el diseño y desarrollo de las programaciones curriculares. Vivir y hacer vivir la democracia en el aula multigrado supone:

1.- Superar el concepto de asimilación de los saberes previamente seleccionados y considerados como los propios y adecuados para la consecución de objetivos curriculares: pero alejados de la realidad, y las necesidades e intereses de los alumnos. En el aula rural no tiene demasiado sentido los contenidos clasificados y ordenados por cursos y edades; "cerrar el aprendizaje de contenidos" destinando un tiempo, un espacio y un material curricular concreto (generalmente un libro de texto) supone limitar al alumno al aprendizaje interdisciplinar y globalizado. La cuestión es ¿porque si un alumno tiene interés y está motivado por un determinado tema y/o contenido se le debe reprimir diciéndole que "no toca" ni ese contenido ni ese tema porque está "cursando" un determinado grado escolar? ... el poder de elegir un tema, de forma libre, si es de interés para el alumno no debe suponer una barrera a su aprendizaje y todavía menos si ese tema es compartido con compañeros que pueden tener la misma edad, ser mayores o menores que uno mismo; éste es el valor pedagógico del aula multigrado, en donde la diversidad y 
el deseo de avanzar contenidos junto con tus compañeros de aula, no supone un problema sino un beneficio pedagógico.

2.- Dimensionar la autonomía de aprendizaje y el autoaprendizaje. Vivir la democracia en el aula supone también ser capaz de aceptar y hacerse cargo de los errores y aciertos como persona responsable de las decisiones que se toman en el desarrollo de las actividades del aula. No todo lo que se hace y como se hace en la clase es aceptado por todos; sin embargo, el alto grado de autonomía de aprendizaje permite al alumno rural argumentar sus decisiones y no tender al conformismo esperando la corrección del maestro o la propuesta de un compañero. La diversidad de edades supone que de forma natural, el aula se convierta en un espacio de autoaprendizaje para todos los alumnos y el maestro.

\section{3.- Concebir las estrategias didácticas como instrumentos o medios válidos y funcionales} para la adquisición de un aprendizaje significativo y democrático; si bien es cierto que la globalización ofrece al alumno un conocimiento integrado frente a la una estructura lógica pero fragmentada de las disciplinas científicas, no es menos evidente que las estrategias didácticas que facilitan este tipo de adquisición de conocimiento (método del proyectos, centros de interés, investigación en el medio, aprendizaje basado en la resolución de problemas, etc.) vienen acompañadas de una finalidad real que orienta los procedimientos de trabajo y les ofrece la oportunidad de afianzar esos procedimientos bajo los parámetros de valores plurales democráticos y vivir el proceso en sí mismo. Escoger mediante el debate y el diálogo, el tema motivo de estudio entre todos los alumnos de un aula multigrado, para el estudio colaborativo de ese tema, implica necesariamente diálogo, respeto hacia las argumentaciones de los "otros" y libertad para poder exponerlas, participación y motivación para llegar a un consenso, y sin duda alguna tomar conciencia de la importancia de igualdad de oportunidades cuando se construye y forma parte de un proyecto común.

\section{4.- Concebir la evaluación como la única forma de tomar conciencia del proceso de} aprendizaje individual y colectivo. La democratización del proceso de evaluación supone utilizar instrumentos abiertos y plausibles para ser discutidos y reelaborados y que permitan revisar las propias actuaciones y las de los demás para inducir mejoras. La participación que conlleva una evaluación de estas características supone necesariamente contemplar el proceso evaluación-aprendizaje-evaluación, en la que la evaluación es 
entendida como en los que la misma evaluación es entendida como una valoración del aprendizaje y la relación asimétrica entre alumnos de distintas edades que aprenden dentro del aula contribuye al desarrollo de modalidades y tipos evaluación en los que participan diferentes agentes y que permiten la sistematización de los aprendizajes por delante de la fiscalización de los mismos. Evaluación en la que el intercambio entre iguales permite dar valor a las preguntas y no solo a las respuestas, facilita la auto-evaluación y la coevaluación; "los "conatos" de autoevaluación y de coevaluación están provocados muchas veces por las propias necesidades del docente de atender a otros alumnos del grupo, que por la creencia en sus posibilidades educativas, se utilizan en las aulas rurales, pero no siempre con intencionalidad evaluadora para la sistematización y valoración del aprendizaje por parte del maestro de todas formas (Abós y Boix,2017, p 47); de todas formas "el docente sigue siendo el principal agente de la evaluación, y aunque el alumnado como agente evaluador está presente en algunas dinámicas en las que se hace referencia a la evaluación entre iguales, ésta queda limitada, en muchas ocasiones, a la corrección de ejercicios en la mayoría de los casos entre alumnado del mismo nivel." (Abós y Boix, 2017, p 47)

5.- Implicar la comunidad educativa y rural en la gestión del centro y del aula. Siguiendo a Dewey (1995) la democracia es un proceso que hay que vivirlo. Para este autor la comunidad y la escuela están íntimamente ligadas y los procesos democráticos de la sociedad y la comunidad se reflejan en la escuela y viceversa. "Una democracia es más que una forma de gobierno; es primariamente un modo de vivir asociado, de experiencia comunicada conjuntamente" (p. 98). La escuela rural es un espacio de empoderamiento para la comunidad rural; Touraine (1994) nos dice que la responsabilidad de la ciudadanía es la base de la democracia, y en ello está el convencimiento de las potencialidades de los miembros de las comunidades rurales y educativas. En un aula multigrado rural la implicación de la comunidad es activo, próximo y por los general, familiar; es un espacio que facilita la labor conjunta entre maestros, alumnos y familias pero también de agentes educativos, sociales comunitarios que forman parte del territorio rural, proyectando la dimensión territorial de la escuela. Este posicionamiento interpela directamente al lugar que la escuela ocupa en el territorio, cuestión nuclear facilitar el desarrollo de proyectos educativos comunes, basados en valores plurales. 


\section{MODELOS PEDAGÓGICOS PARA VIVIR LA DEMOCRÁCIA: EJEMPLIFICACIONES DE ACTIVIDADES Y PROYECTOS DE ESCUELA RURAL DEMOCRÁTICA}

$\mathrm{Si}$, como venimos diciendo, entendemos la sociedad como el espacio donde se establecen relaciones, más o menos complejas, entre los distintos individuos que la forman, estás relaciones construyen las normas de funcionamiento de la comunidad que, como si se tratara de un circuito cerrado, terminan marcando las relaciones entre los individuos. La flexibilidad de las relaciones puede también templar las normas sociales y viceversa, garantizando el éxito colectivo que es tener una sociedad sana y democrática donde todos los individuos se sientan parte activa de la misma.

Si comprendemos, además, que la escuela es un reflejo de la sociedad, entenderemos que las relaciones entre sus miembros marcaran las normas de funcionamiento del centro, creando el mismo circuito cerrado. Incidir en estas relaciones (alumnado-alumnado, alumnado-maestros, maestros-maestros, alumnado-entorno, maestros-entorno) y flexibilizarlas es primordial para alcanzar el éxito colectivo que es el aprendizaje. En el caso de las escuelas rurales, la incidencia es más directa ya que se trata de comunidades más pequeñas en número de miembros, pero no por ello menos complejas, aunque por lo general son más flexibles.

En este entorno de aula como patrón de la sociedad en la que se inserta la escuela, los modelos pedagógicos que dan cabida a los valores plurales y facilitan vivir la democracia se enmarcan en un contexto didáctico o bien mixto o bien activo-participativo, en ningún momento en la práctica pedagógica del aula multigrado con una perspectiva de enseñanza tradicional; este tipo de práctica no permite vivir la democracia, y en consecuencia, no contempla una educación democrática sustentada sobre los principios básicos de la democratización participativa. El modelo pedagógico mixto que combina elementos de la didáctica tradicional con elementos de la didáctica activo-participativa permite sostener, en el desarrollo de sus estrategias didácticas, relaciones horizontales y verticales entre los miembros del aula (maestros y alumnos, y alumnos-alumnos de distintas edades y /o de las mismas edades) que justifican por si mismas la necesidad de trabajar los valores plurales democráticos para el propio mantenimiento de la convivencia; de la misma forma que lo promueve el modelo pedagógico activo-participativo, en donde el principal protagonista es el alumno, no el libro de texto, y en donde las relaciones entre 
los miembros del aula suelen ser básicamente horizontales y donde se flexibilizan también, junto con el modelo mixto, las actuaciones para que los aprendizajes fluyan de forma natural y en donde se aprovecha la diversidad como herramienta de cambio, reforzando así las relaciones entre los individuos del grupo.

\section{EJEMPLIFICACIONES ACTIVIDADES Y PROYECTOS}

Para mostrar la parte práctica de lo que se viene relatando, se presentan unas ejemplificaciones de actividades que pretenden ser una guía para utilizar en las aulas multigrado donde la diversidad de edades y necesidades obliga a las escuelas a replantearse modelos tradicionales, basados únicamente en la transmisión de conocimientos a través de libros de texto donde se segmentan de forma arbitraria los contenidos a tratar en cada etapa. Analizar qué necesita el grupo-clase y adaptar las actividades a estas necesidades es muy importante. Para conseguir este objetivo necesitamos un enfoque activo, donde el maestro no es un mero transmisor de conocimiento o distribuidor de tareas, sino que es un miembro también activos de la comunidad escolar, que se relacionan con el alumnado y propician situaciones de auto-aprendizaje para alumnos.

El maestro debe poner a disposición del alumnado todos los recursos posibles para que estos aprendizajes circulen, y dentro de estos recursos también pueden encontrarse los libros de textos si la práctica pedagógica parte de un modelo mixto. Estos materiales utilizados de forma puntual son útiles en un aula multigrado, pero lo que no se puede pretender es que se conviertan en el centro de la vida educativa de la escuela y en el hacer vivir la democracia.

Todas las actividades que se describen a continuación están diseñadas para ser el máximo de flexibles y adaptarse a distintos contextos previo análisis de las necesidades del grupo donde se va a desarrollar.

\begin{tabular}{|c|c|c|}
\hline IVIDAD & AREA & OBJETIVOS \\
\hline \multirow{3}{*}{$\begin{array}{l}\text { LIBRETA DE } \\
\text { LA } \\
\text { IMAGINACIÓN }\end{array}$} & Lengua & \multirow{3}{*}{$\begin{array}{l}\text { - Conocer y aplicar distintas tipologías } \\
\text { textuales. } \\
\text { - Conocer y aplicar distintas normas } \\
\text { ortográficas. } \\
\text { - Ser consciente de los propios errores para } \\
\text { poder corregirlos. }\end{array}$} \\
\hline & VALORES & \\
\hline & $\begin{array}{c}\text { Participación } \\
\text { Libertad } \\
\text { Diálogo }\end{array}$ & \\
\hline
\end{tabular}




\begin{tabular}{|c|c|}
\hline & $\begin{array}{l}\text { - Potenciar la creatividad del alumnado. } \\
\text { - Potenciar el uso del lenguaje asertivo. } \\
\text { - Respetar las propias producciones y las } \\
\text { de los demás. }\end{array}$ \\
\hline & MARCO METODOLÓGICO EN UN AULA MULTIGRADO \\
\hline & $\begin{array}{l}\text { Esta actividad se puede situar en el proceso de adquisición de la } \\
\text { lectoescriptura y se basa en la generación de aprendizajes a partir de las } \\
\text { propias producciones del alumnado y en los aprendizajes colectivos, algo } \\
\text { primordial en aulas multigrado. } \\
\text { Basarse en modelos propios ayuda a los niños y niñas a partir de una } \\
\text { realidad más cercana y a empatizar con los textos motivándolos a leer y a } \\
\text { escribir ya sean producciones propias o las de sus compañeros y } \\
\text { compañeras. Además, el hecho de participar en la toma de decisiones sobre } \\
\text { los criterios de evaluación les implica en todas las fases del proceso y hace } \\
\text { que se sientan parte del mismo, así como responsabilizarlos de sus } \\
\text { decisiones. Por otra parte, al conocer de antemano los criterios, saben } \\
\text { exactamente qué se les pide y ayuda a la auto-regulación, ya que el } \\
\text { alumnado quien detecta los errores y buscan la manera de corregirlos. } \\
\text { Durante este proceso se generan aprendizajes significativos porqué nacen de } \\
\text { ellos mismos y estimulan herramientas que más tarde podrán utilizar en } \\
\text { otras situaciones: busca de información, análisis de un texto, ampliación de } \\
\text { vocabulario,... }\end{array}$ \\
\hline & FUNCIONAMIENTO EN UN AULA MULTIGRADO \\
\hline & $\begin{array}{l}\text { Cada alumno/a dispone de una libreta propia, personalizada. En ella podrán } \\
\text { escribir, pegar o dibujar todo lo que les apetezca. En un primer momento, se } \\
\text { pactan con todo el grupo los criterios que se deberán seguir para usar y } \\
\text { avaluar la "Libreta de la imaginación": coherencia, adecuación, } \\
\text { presentación, cohesión, léxico, ortografia,... } \\
\text { El maestro o la maestra guiará el debate en función de los criterios } \\
\text { establecidos por el claustro y se acabará editando un documento con los } \\
\text { criterios acordados por el grupo (fig. l). El documento se pegará en un lugar } \\
\text { visible i accesible de la libreta. Estos criterios se utilizaran para elaborar una } \\
\text { rúbrica (fig. 2) con la que el alumnado evaluará cada una de sus } \\
\text { producciones. } \\
\text { Semanal o quincenalmente se les puede proponer una tipología textual } \\
\text { distinta, previa presentación del modelo, que tendrán que escribir en la } \\
\text { libreta siguiendo los criterios establecidos. } \\
\text { Al principio de cada sesión cada niño y niña leerá su producción delante del } \\
\text { resto del grupo. Al terminar el resto de compañeros y compañeras, } \\
\text { oralmente, pueden formularle algún aspecto a mejorar, empezando siempre } \\
\text { por algún aspecto positivo relacionado con el texto o con la lectura del } \\
\text { mismo. El niño o niña que ha leído puede rebatir las aportaciones de sus } \\
\text { compañeros y compañeras. } \\
\text { Al finalizar de leer todos los niños y niñas cada uno hará su rúbrica y } \\
\text { valorará finalmente la globalidad del texto escrito. Otra forma de hacerlo es } \\
\text { que otro compañero o compañera haga la rúbrica, argumentando después al } \\
\text { alumno propietario de la libreta el porqué de su valoración. } \\
\text { Finalmente el maestro o la maestra animará a los niños y niñas a corregir } \\
\text { (mejorar) los textos en función de la valoración de la rúbrica. } \\
\text { Durante el tiempo que no se usen las "Libretas de la imaginación" pueden } \\
\text { estar expuestas y a disposición de todos para ser usadas como libro de } \\
\text { lectura. }\end{array}$ \\
\hline
\end{tabular}




\section{¿QUÉ TENGO QUE HACER PARA SER UN/A EXPERTO/A?}

- Que no haya ninguna falta.

- Que la letra esté muy recta.

- Que la letra sea muy clara.

- Que siempre haya la fecha.

- Que siempre haya el título.

\section{¿QUÉ TENGO QUE HACER PARA SER UN/A AVANZADO/A?}

- Que hay una o dos faltas:

- Que ponemos un punto al final de la frase.

- Que cuando empezamos a escribir o después de un punto escribimos letra de palo.

- Que ponemos mayúscula cuando escribimos el nombre de una persona, de un pueblo, de una ciudad o de un sitio.

- Que la letra entienda.

- Que las palabras estén rectas.

- Que los textos tengan título.

- Que las palabras estén separadas.

- Que ponemos la fecha de cuando escribimos el texto.

- Que dejamos un margen.

- Que escribimos lo que se nos pide.

\section{¿QUÉ TENGO QUE HACER PARA SER UN/A APRENDIZ?}

- Que hay más de dos faltas y hasta seis.

- Si nos dejamos tres de las cosas que deberíamos hacer para que la libreta estuviera bien.

\section{¿QUÉ TENGO QUE HACER PARA SER UN/A NOVEL?}

- Que haya más de seis faltas.

- No hacemos nada de las cosas que deberíamos hacer para que la libreta estuviera a un nivel de avanzado.

- Si no hacemos la libreta de la imaginación. 


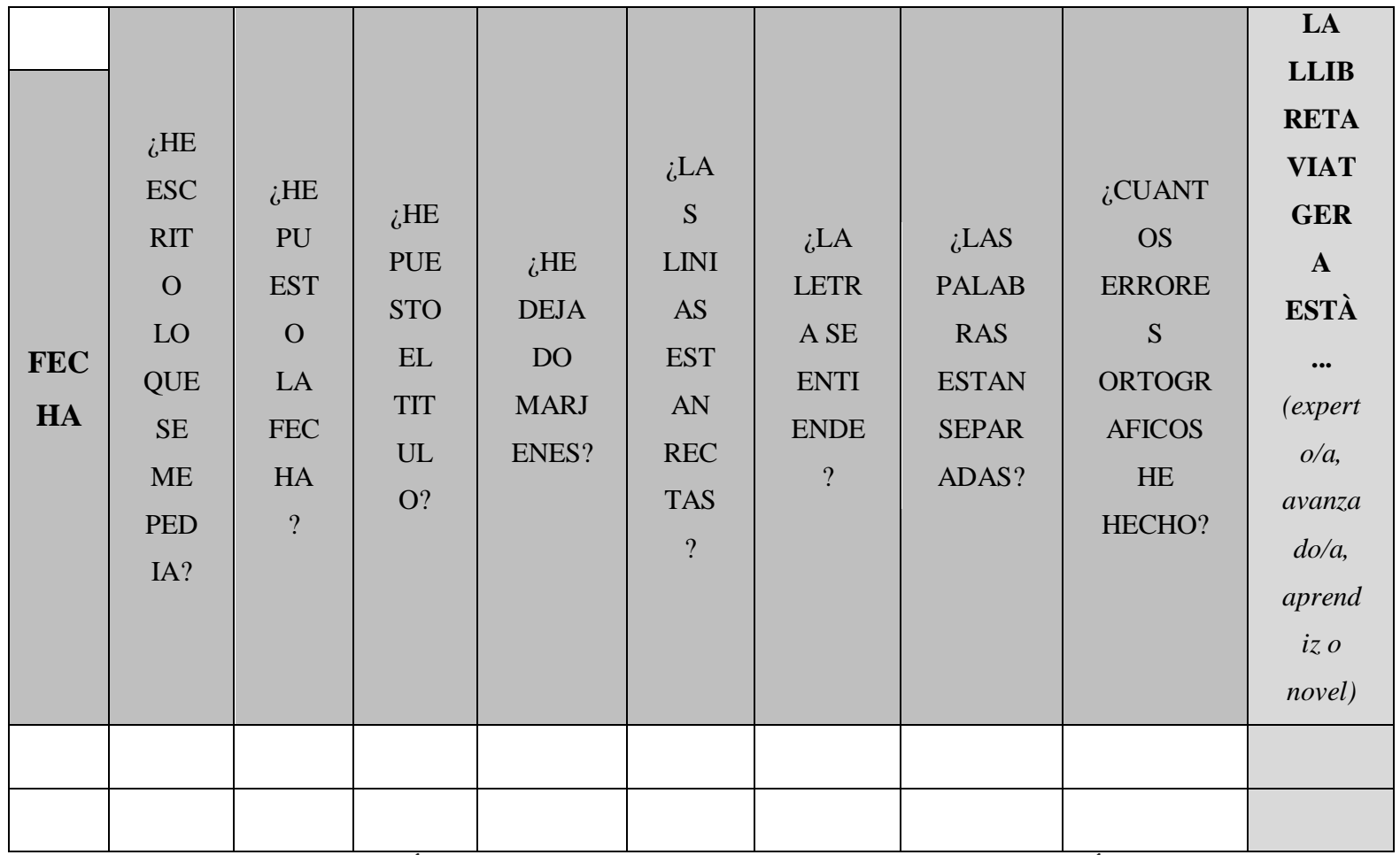

Figura. 2: EJEMPLO DE RÚBRICA DE LA LIBRETA DE LA IMAGINACIÓN

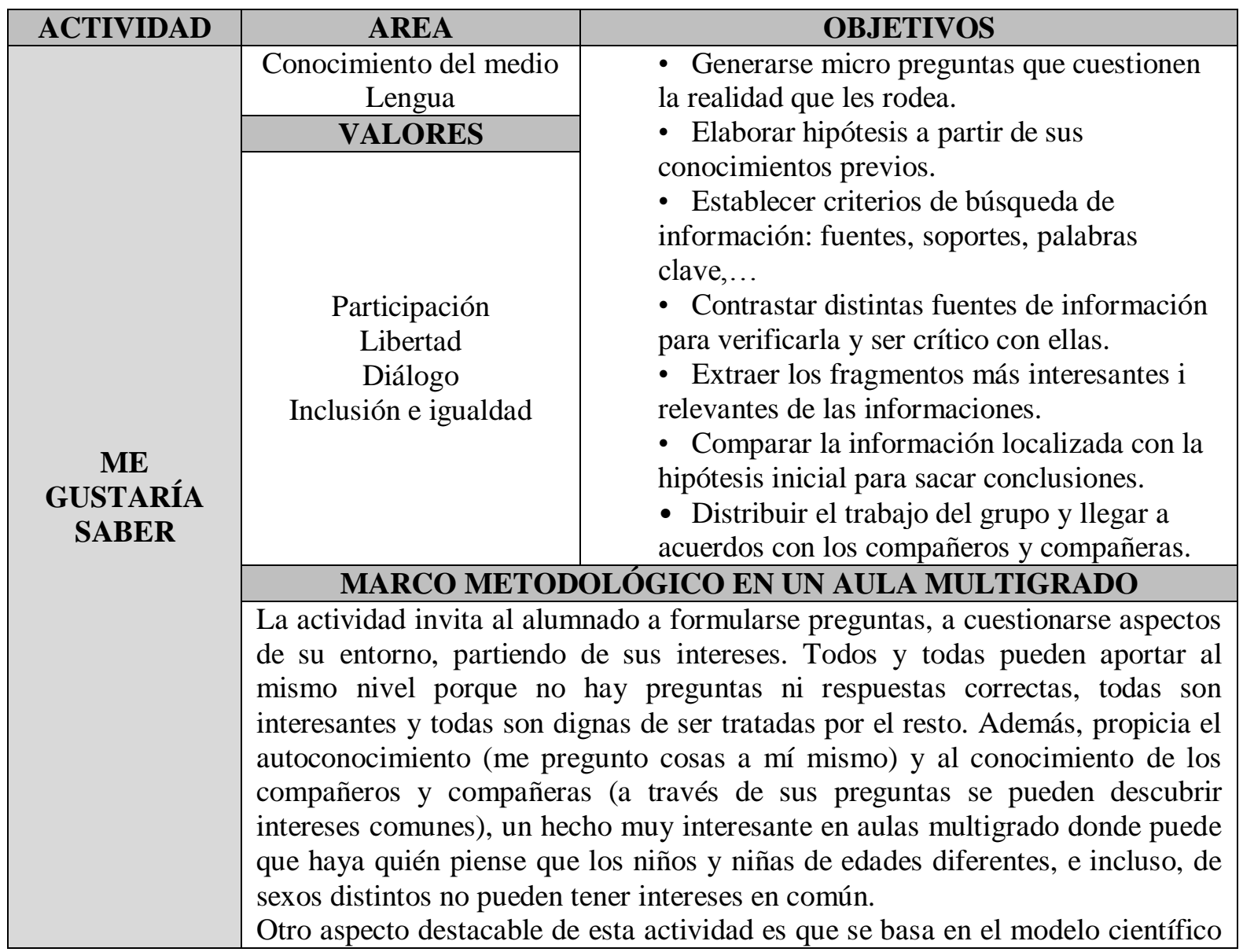


$\mathrm{y}$ en las competencias informacionales, es decir que el trabajo gira entorno en demostrar que una hipótesis es correcta o no. Para hacerlo es necesario activar distintas herramientas que permitan elaborar criterios de búsqueda, adaptarlos a las necesidades, discriminar la información esencial, relaborarla y mezclarla con conocimientos previos para crear conocimiento nuevo y finalmente comunicarlo de forma efectiva.

Todas estas herramientas permiten resolver una gran cantidad de situaciones que se irán encontrando a lo largo de la vida. Son competencias aplicables a muchos trabajos distintos de campos muy diversos, des de los más científicos y tecnológicos a los más relacionados con las humanidades.

\section{FUNCIONAMIENTO EN UN AULA MULTIGRADO}

En un espacio del aula habrá un mural con el título de la actividad. En él, los niños y niñas podrán pegar trozos de papel con las preguntas que se les vayan ocurriendo. Todas son válidas la única condición es que estén escritas correctamente. La preguntas se pueden escribir al llegar a la clase por la mañana o después del recreo (momento en el que han podido observar muchas aspectos del entorno: hojas, plantas, insectos, piedras,...).

Durante la sesión que dure la actividad en grupos de dos o tres podrán escoger una de las preguntas que hay en el mural (puede ser la suya o la de otro compañero/a) y empezaran a redactar el informe (fig. 3). Primero tendrán que ponerse de acuerdo con la hipótesis, argumentando las distintas ideas que pueda haber en el grupo. Después escogerán la forma de comprobar la hipótesis. Como puede haber preguntas sobre aspectos muy diferentes, las posibilidades de comprobación también son muy diversas. Por eso es importante animar al alumnado a ser creativo y a buscar maneras motivadoras enfocadas a experimentar.

Hayan escogido la forma que sea, será necesario buscar información: de qué tipo, dónde, qué palabras,... Es importante que el alumnado reflexione esta parte del proceso ya que marcará los avances en su investigación. Cuestionarles y hacerles argumentar sus decisiones les ayudará.

Una vez hecha y visada esta primera parte, empieza la búsqueda en sí. Tener una biblioteca ordenada y adaptada a sus necesidades (sea en el aula o en otro espacio del colegio) es básico para garantizar el éxito de la actividad. En esta parte del proceso podemos mostrarles los apartados de un libro que facilitaran la búsqueda: índices, glosarios,... Cuando localicen una información interesante (palabras, frases, párrafos, dibujos, esquemas,...) pueden copiarla en el informe o fotocopiarla i pegarla en el informe, apuntando la fuente de dónde la han sacado.

En el caso de Internet (muy tentador) es necesario establecer criterios para poder desechar las entradas que no interesen. Por ejemplo: si están usando un buscador, en el que han puesto unas palabras clave, entre las diez primeras entradas deben encontrar (sin abrirlas) alguna información que les pueda ayudar en la investigación. Si no es así deberán cambiar las palabras clave. Cuando encuentren una entrada interesante (una o dos como máximo) pueden abrirla en una pestaña aparte y revisar lo que hay en ella. Si encuentran alguna información que les pueda servir se tratará de imprimirla o copiarla en el informe.

Cuando el grupo considere que ya ha recogido todo lo necesario para comprobar su hipótesis, puede empezar el análisis de las informaciones mezclándolas entre ellas y con sus conocimientos previos hasta crear una nueva información, un nuevo discurso en el que emplearan sus propio vocabulario. Llegados a este punto ya pueden comparar el nuevo discurso con la hipótesis para comprobar su veracidad. 
Otra forma de terminar la actividad es comunicando esta información al resto de compañeros y compañeras a través de un periódico escolar, del blog del colegio, de un mural, de una infografía,...

Figura 3. EJEMPLO DE INFORME

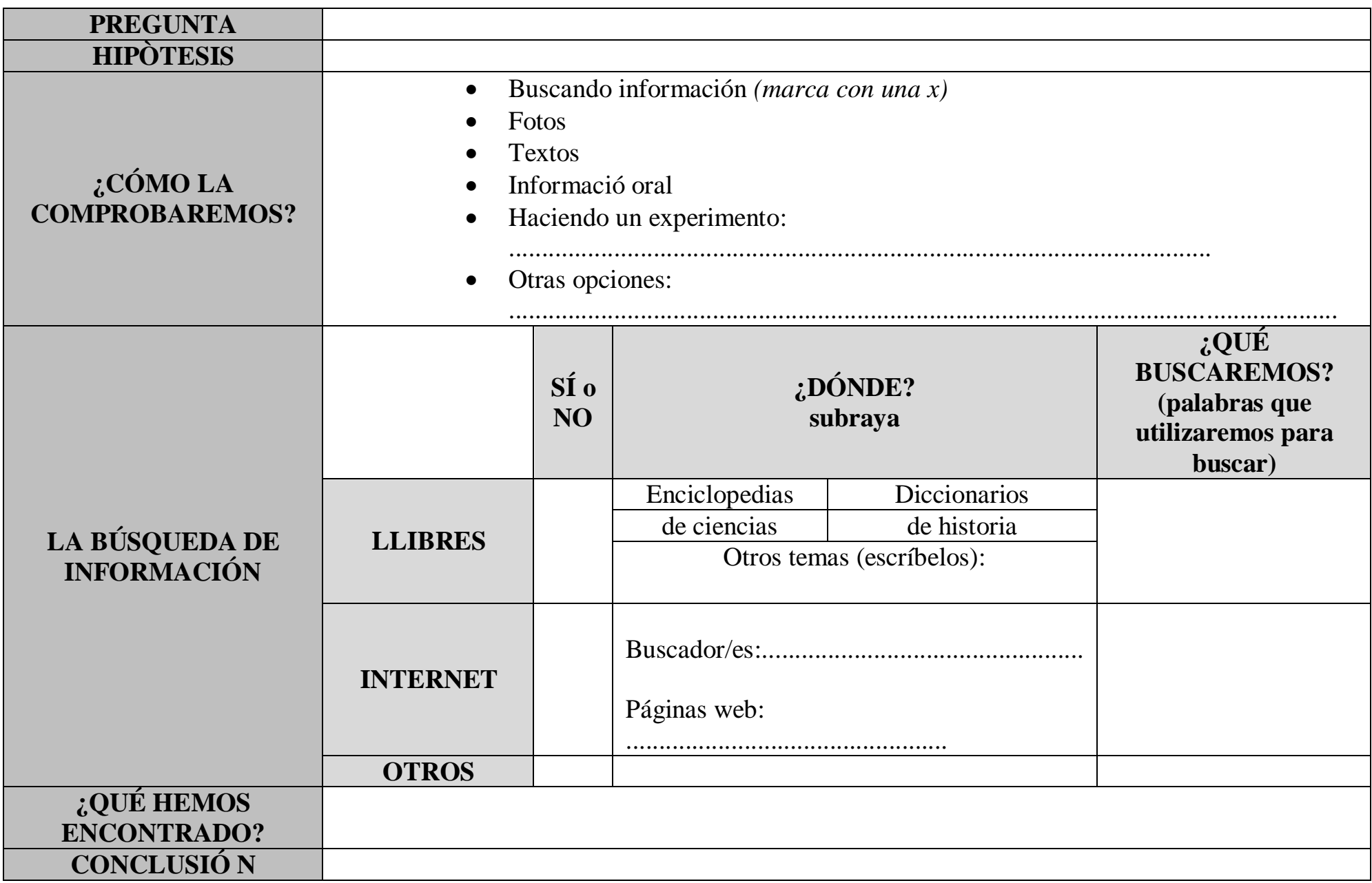

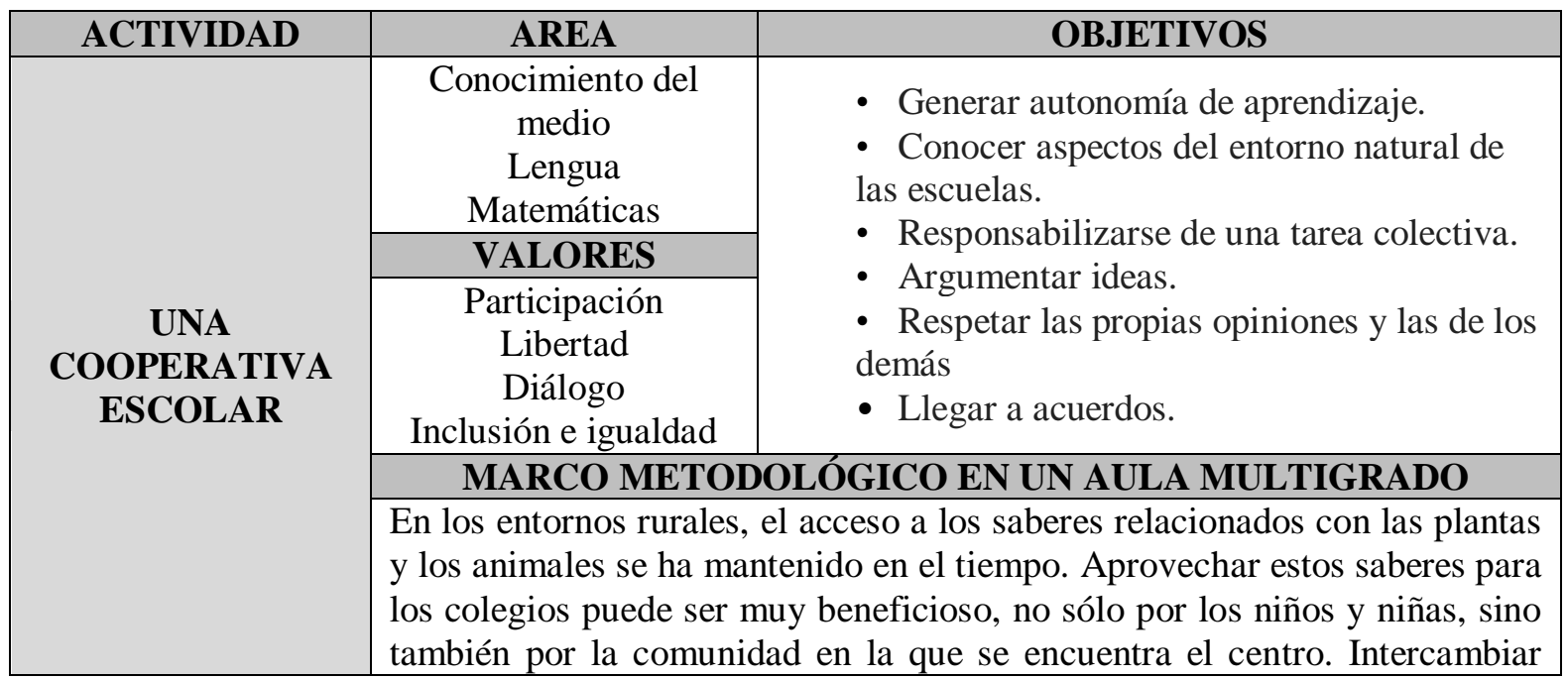


conocimientos entre distintas generaciones fortalece en vínculo entre ellas, siendo la escuela el punto de encuentro.

En esta actividad se propone un objetivo común y compartido entre todo el alumnado del centro que parte de una organización horizontal, es decir que todos y todas (alumnado y profesorado, tengan la edad que tengan) son iguales desempeñando una tarea que beneficia a la comunidad. Estas tareas tienen a ver, también, con los intereses y las capacidades de cada uno. La cooperativa no se trata de un juego simbólico, es una forma de poner en práctica los aprendizajes o crear nuevos a partir de la realidad, a partir de la manipulación y la experimentación. En definitiva que pone en marcha una gran diversidad de competencias, de herramientas que les permitirán abordar gran variedad de situaciones en el futuro.

Es el mismo alumnado el que lidera el proyecto, el que toma las decisiones y las ejecuta, valorando los errores para buscar alternativas que mejoren el producto o el servicio que ofrecen a su comunidad (familias o vecinos).

\section{FUNCIONAMIENTO EN UN AULA MULTIGRADO}

La cooperativa es una forma de empresa creada y dirigida por el alumnado de todo el centro que vende productos del huerto escolar y/o del gallinero. Este es un ejemplo pero la cooperativa puede vender y ofrecer todo tipo de productos y servicios, en función de los intereses de los niños y niñas.

En primer lugar hace falta redactar un documento en el que se explique a qué se dedica la cooperativa y su funcionamiento (asamblea, derechos y deberes de los socios y las socias, funciones de los equipos de trabajo, cargos ejecutivos, en qué se invierten los excedentes,...). En la red hay muchos sitios orientados a la creación de cooperativas escolares donde podrán aclarar las dudas que tengáis al respeto.

Los equipos de trabajo son la base del funcionamiento de la cooperativa ya que cada uno se dedica a una tarea distinta, pero necesaria para conseguir vender los productos. Si se sigue en el ejemplo de la cooperativa dedicada a la venta de productos del huerto y del gallinero se pueden tener estos equipos de trabajo:

- Huerto: encargado del cuidado de las plantas, de su crecimiento y recolección.

- Gallinero: encargado del cuidado de las gallinas y de la recolección de huevos.

- Tesorería: encargado de la gestión económica de la cooperativa y de los distintos equipos de trabajo.

- Comunicación: encargado de informar a los clientes de los productos que se ofrecen y de la gestión de los pedidos.

- Tienda: encargada de empaquetar, entregar y cobrar los productos.

Es importante abrir cada sesión con lo que ha quedado pendiente de la anterior y que sean los niños y niñas los que prioricen las actuaciones que se llevaran a cabo. Por lo tanto es necesario que dentro de cada equipo de trabajo haya una organización de tareas y que cada miembro tenga clara su función.

Igualmente los procesos de toma de decisiones tienen que ser inclusivos, escuchando todas las propuestas y votando para llegar a acuerdos amplios. 


\begin{tabular}{|c|c|c|}
\hline ACTIVIDAD & AREA & \multirow{4}{*}{$\begin{array}{l}\text { - Generar autonomía de trabajo. } \\
\text { - Adaptarse a los diferentes niveles y ritmos de } \\
\text { aprendizaje. } \\
\text { - Respetar la diversidad. } \\
\text { - Autoregularse. }\end{array}$} \\
\hline \multirow{7}{*}{$\begin{array}{c}\text { PLANES } \\
\text { DE } \\
\text { TRABAJO }\end{array}$} & & \\
\hline & VALORES & \\
\hline & $\begin{array}{c}\text { Participación } \\
\text { Libertad } \\
\text { Diálogo } \\
\text { Inclusión e igualdad }\end{array}$ & \\
\hline & \multicolumn{2}{|c|}{ MARCO METODOLÓGICO EN UN AULA MULTIGRADO } \\
\hline & \multicolumn{2}{|c|}{$\begin{array}{l}\text { En un aula multigrado la diversidad de niveles y de necesidades del alumnado es } \\
\text { muy grande y poder prestar atención a cada uno de los miembros del grupo en el } \\
\text { grado adecuado es muy importante. } \\
\text { Los planes de trabajo se basan en potenciar la autonomía de aprendizaje del } \\
\text { alumnado ofreciéndoles la posibilidad de adaptarse a su ritmo de trabajo y a sus } \\
\text { necesidades. El hecho de que cada uno tenga tareas distintas potencia el respeto a } \\
\text { la diversidad y, además, abre la puerta a que se presten ayuda entre ellos y ellas. } \\
\text { El lenguaje que utilizan los niños y niñas resulta más entendible y por lo tanto } \\
\text { ayuda a la consolidación de los aprendizajes. Por otra parte, el alumno o alumna } \\
\text { que está prestando la ayuda tiene que utilizar unas herramientas para comunicar, } \\
\text { cose que le obliga a generar unas estructuras mentales que también consolidan } \\
\text { estos aprendizajes. } \\
\text { Se puede decir que con esta dinámica de trabajo se construyen aprendizajes } \\
\text { compartidos y la gestión del tiempo es mucho más productiva que utilizando } \\
\text { metodologías magistrales donde el maestro o la maestra eran los generadores } \\
\text { únicos de contenidos. }\end{array}$} \\
\hline & \multicolumn{2}{|c|}{ FUNCIONAMIENTO EN UN AULA MULTIGRADO } \\
\hline & \multicolumn{2}{|c|}{$\begin{array}{l}\text { Cada niño y cada niña dispondrá de una carpeta con el material necesario para } \\
\text { realizar el plan de trabajo: cuaderno, fichas,... El alumnado será el responsable } \\
\text { de este material y habrá un espacio en el aula donde se guardará. } \\
\text { Al principio de la sesión el maestro o la maestra presentará todos los materiales } \\
\text { que se van a utilizar por primera vez, por ejemplo si se jugará a un juego de mesa } \\
\text { se explicaran el funcionamiento básico del mismo. Después entregará el plan de } \\
\text { trabajo (fig. 4) e indicará cuantas sesiones se van a dedicar a ello para que los } \\
\text { niños y niñas puedas gestionar el tiempo. } \\
\text { El alumnado podrá empezar por la actividad que desee, a no ser que alguna } \\
\text { requiera un número máximo o mínimo de participantes. En tal caso tendrán que } \\
\text { llegar a un acuerdo para resolver el conflicto. } \\
\text { A medida que acaban una actividad, cada alumno/a marcará un cruz en el } \\
\text { apartado "HECHO" y le entregará la tarea al maestro/a. Éste/a la revisará, si } \\
\text { puede ser conjuntamente con el niño o la niña, marcará el apartado } \\
\text { "REVISADO" y anotará en el apartado "OBSERVACIONES" aquellos aspectos } \\
\text { a corregir o mejorar. Una vez el alumno/a haya corregido estos aspectos y el } \\
\text { maestro/a los haya vuelto a revisar, se le podrá un sello que escogerá el niño o la } \\
\text { niña. Después de esto podrá cambiar de actividad y hacer la siguiente tarea del } \\
\text { plan de trabajo. } \\
\text { Este proceso se puede producirse durante la misma sesión o el maestro/a puede } \\
\text { revisar las tareas en otro momento. También se pueden proponer actividades } \\
\text { autocorrectivas en las que los mismos niños y niñas marquen el apartado } \\
\text { "REVISADO" i por lo tanto se autoregulen. } \\
\text { Es importante empezar con pocas actividades en las que el alumnado se sienta } \\
\text { seguro y cómodo. Poco a poco se pueden introducir actividades más complejas y } \\
\text { más autónomas. }\end{array}$} \\
\hline
\end{tabular}


Fig. 4: EJEMPLO DE PLAN DE TRABAJO DE MATEMÁTICAS

\begin{tabular}{|c|c|c|}
\hline ACTIVIDAD & AREA & \multirow{4}{*}{$\begin{array}{l}\text { OBJETIVOS } \\
\text { - Dinamizar el aprendizaje de la lectura } \\
\text { entre iguales. } \\
\text { - Potenciar los aprendizajes referidos a } \\
\text { habilidades y estrategias lectoras. } \\
\text { - Mejorar la expresión oral. } \\
\text { - Crear contextos de aprendizaje } \\
\text { significativos y motivadores. } \\
\text { - Generar experiencias de lectura } \\
\text { positivas y placenteras o disfrutar con la } \\
\text { lectura. } \\
\text { - Establecer lazos afectivos entre } \\
\text { padrinos y ahijados en torno a la lectura. }\end{array}$} \\
\hline \multirow{7}{*}{$\begin{array}{l}\text { APADRINAMIENTO } \\
\text { LECTOR }\end{array}$} & Lengua & \\
\hline & VALORES & \\
\hline & $\begin{array}{c}\text { Participación } \\
\text { Libertad } \\
\text { Diálogo } \\
\text { Inclusión e igualdad }\end{array}$ & \\
\hline & \multicolumn{2}{|c|}{ MARCO METODOLÓGICO EN UN AULA MULTIGRADO } \\
\hline & \multicolumn{2}{|c|}{$\begin{array}{l}\text { Los apadrinamientos lectores son una dinámica basada en la tutoría } \\
\text { entre iguales, es decir que no interviene un adulto de forma directa } \\
\text { Con esto se consigue un beneficio mutuo entre el padrino/a (mayor o } \\
\text { con más experiencia) y el ahijado/a (menor o con menos experiencia) } \\
\text { ya que por una parte el padrino/a debe dominar no sólo la lectura sino } \\
\text { una serie de estrategias orientadas a ayudar a su ahijado a mejorar. Al } \\
\text { hacer este proceso aumenta también su grado de mejora. Y no sólo eso } \\
\text { sino que en aquellos casos que haya alguna dificultad, el hecho de ser } \\
\text { padrino/a hace crecer la autoestima i mejor el autoconcepto. } \\
\text { Por otra parte, el ahijado es acompañado por un/a compañero/a que } \\
\text { posiblemente utilizará unas estrategias que les serán más cercanas. Es } \\
\text { por esta cercanía y por el vínculo que se puede crear entre la pareja } \\
\text { que genera un ambiente idóneo para la mejora de la lectura. } \\
\text { Esta actividad es ideal para un aula multigrado ya que organiza y y } \\
\text { dinamiza la diversidad que puede haber. Aprovecha esta diversidad en } \\
\text { beneficio del grupo. }\end{array}$} \\
\hline & \multicolumn{2}{|c|}{ FUNCIONAMIENTO EN UN AULA MULTIGRADO } \\
\hline & \multicolumn{2}{|c|}{$\begin{array}{l}\text { Antes de empezar el apadrinamiento es necesario que los padrinos/as } \\
\text { hagan unas sesiones preparatorias en las que se pongan en la piel del } \\
\text { padrino/a y del ahijado/a. Estas sesiones servirán para compartir } \\
\text { estrategias como: } \\
\text { - Antes de empezar la lectura mirar los elementos del } \\
\text { libro y hacer deducciones a través de los dibujos y/o el título. } \\
\text { - Respetar el ritmo y el nivel de lectura del ahijado/a. } \\
\text { inferenciales,...) al terminar un párrafo o un capítulo. } \\
\text { - Hacer una lectura expresiva. } \\
\text { Las parejas lectoras estarán elaboradas por el equipo de maestros/as } \\
\text { teniendo en cuenta los niveles de lectura de padrino/a y ahijado/a, y } \\
\text { asegurando que se pueden entender. } \\
\text { Al terminar la fase preparatoria, el acto de presentación de las parejas } \\
\text { se puede hacer con toda solemnidad para remarcar el valor de la } \\
\text { dinámica. En este acto, que puede empezar con algún juego para }\end{array}$} \\
\hline
\end{tabular}




\begin{tabular}{|l|l|}
\hline romper el hielo, se les entregará un carnet con la foto de los dos, se les \\
entregará el libro de apadrinamiento (fig. 5) y firmarán el compromiso. \\
Después de eso podrán escoger la primera lectura. \\
Es importante que lectura se desarrolle en un lugar donde estén \\
cómodos y el ambiente sea tranquilo: la biblioteca, una aula con \\
cojines o sillones, en el patio o jardín,... \\
Al terminar la sesión los/las dos valorarán cómo ha ido teniendo en \\
cuenta los criterios establecidos en el informe (fig. O). Estos criterios se \\
pueden acordar conjuntamente con los niños y niñas y pueden servir a \\
modo de rúbrica.
\end{tabular}

Fig. 5: EJEMPLO DE LIBRO DE APADRINAMIENTO 

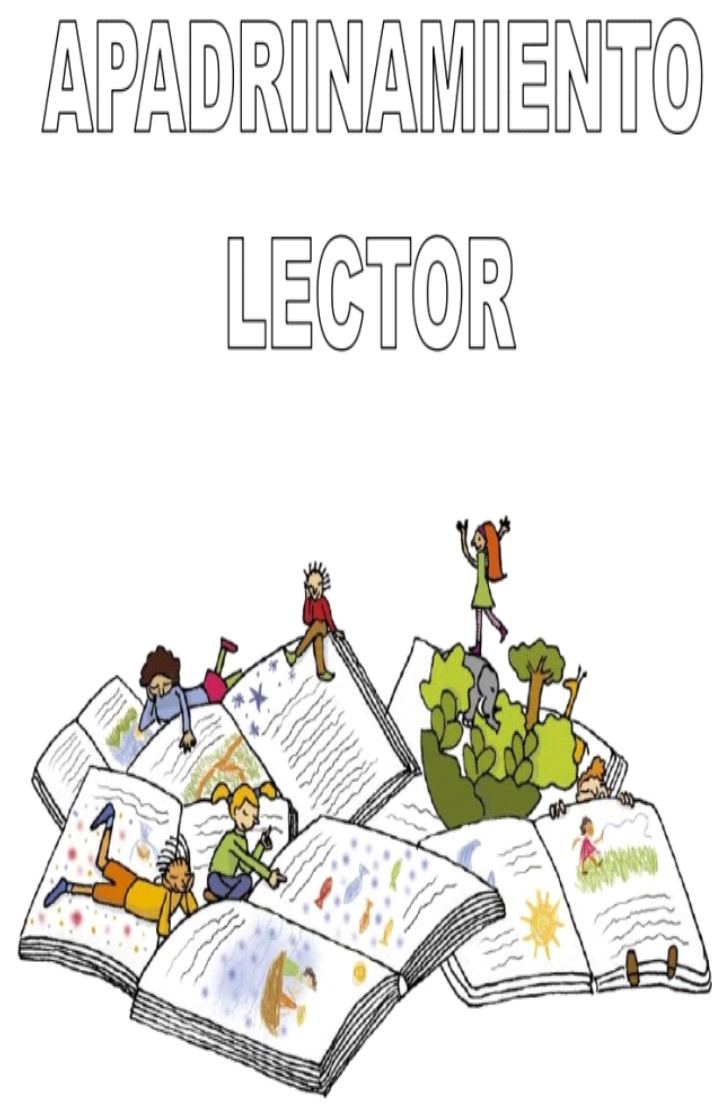

Ahijado/a:

Padrino/a:

Fig. 5: EJEMPLO DE INFORME DE LA SESIÓN

\section{Compromiso de apadrinamiento}

Yo, el padrino/a de lectura de , me comprometo a ser para ayudarlo / la a leer mejor cada día y para disfrutar juntos de la magia de la lectura! Por eso, siempre me prepararé muy bien las lecturas.

Y por eso, firmo estos compromisos.

Firma del padrino / a:

Y yo, me comprometo a

seguir los consejos de mi padrino / a para aprender a leer y comprender cada vez mejor las fantásticas historias de todos los libros que compartimos.

Y por eso, firmo este compromiso.

Firma del ahijado / a: 


\section{Informe de la sesión \\ Fecha: \\ Título del libro leído:}

\section{Padrino/a}

\begin{tabular}{|l|l|}
\hline El ahijado/a ha leído bien. (si es que lee) \\
\hline El ahijado/a ha entendido lo que leía. & \\
\hline Me he esforzado a la hora de leer. & \\
\hline He hecho buenas preguntas. & \\
\hline
\end{tabular}

\section{Informe de la sesión}

Ahijado/a

\begin{tabular}{|l|l|}
\hline & \\
\hline El/la padrino/a ha leído bien (entonación, \\
ritmo...)
\end{tabular}

¿Hemos acabado el libro? Sí / NO

Firmas

Para terminar, y siguiendo a Apple y Beane (1999), “debemos tener en cuenta que se concibe una escuela democrática como un escenario que por su estructura y funcionamiento supone un ejercicio práctico de la democracia porque ya su mismo currículum implica oportunidades continuas de explorar las cuestiones que afectan a sus integrantes, de imaginar respuestas a los problemas y de guiarse por ellas". (p. 120). Todos los miembros que forman parte de la escuela han de sentir que la escuela también les pertenece a ellos, es suya, los reconoce, los valora, los apoya, les exige, y 
especialmente una escuela en la que pueden participar, hacerse escuchar, asumir responsabilidades, ser bien tratados por todos, indiferentemente de la edad y teniendo la ocasión de vivir valores como la solidaridad, la tolerancia, la justicia, la igualdad de oportunidades. "La diversidad de tareas y metodologías, de apoyos, ayudas y cuidado, de materiales y recursos didácticos, desde los tradicionales a los que ahora hacen posible las nuevas tecnologías, así como la posibilidad de seguir y apoyar sobre la marcha el ritmo de cada cual, son caminos a transitar en esa dirección y, entonces, retos de la mejora e inclusión" (Escudero, 2012, p 118) y de la consecución de una escuela rural democrática.

\section{REFERENCIAS}

ABÓS, P.; BOIX, R. Evaluación de los aprendizajes en escuelas rurales multigrado. Universidad de Oviedo: Aula, n. 45, p.41-48, 2017.

APPLE, M; BEANE, J.A. Escuelas democráticas. Editorial Morata: Madrid, 1999.

ASAMBLEA GENERAL DE LAS NACIONES UNIDAS. La Declaración Universal de derechos humanos. Paris, Naciones Unidas, 1948.

BUSTOS, A. Escuelas rurales y educación democrática. La oportunidad de la participación comunitaria. Revista Electrónica Interuniversitaria de Formación del Profesorado, 14 (2), pp. 105-114, 2011.

DEWEY, J. Democracia y educación: una introducción a la filosofía de la educación. Ediciones Morata: Madrid, 1995.

DEWEY, J. El niño y el programa escolar. Buenos Aires: Losada, 1972

DEWEY, J. Experiencia y Educación. Buenos Aires: Losada, 1967

ESCUDERO, J.M. La educación inclusiva, una cuestión de derecho. Educatio Siglo XXI: Facultad Educación de la Universidad de Murcia, Vol. 30, nº 2, pp. 109-128, 2012.

FREIRE, P. La educación como práctica de la libertad. Ed: Siglo XXI. Buenos Aires, 1974 “

FROMM, E. El miedo a la libertad. Buenos Aires: Paidós, 2005.

MARTÍNEZ, A. (Coord.) Vivir la democracia en la escuela. Instrumentos para intervenir en el aula y en el centro. Sevilla: MCEP-Kikiriki, (2002).

PIAGET, J. Le droit à l'éducation dans le monde actuel. París: Librairie du Recueil Sirey, 1949. 
ROUSSEAU, J:J. El contrato social.Madrid: Akal, 2017

RUIZ, G. La teoría de la experiencia de John Dewey: significación histórica y vigencia en el debate teórico contemporáneo. Foro de Educación, 11(15), pp. 103-124. 2013. doi: http://dx.doi.org/10.14516/fde.2013.011.015.005

TOURAINE, A. ¿Qué es la democracia? México: Fondo de Cultura Económica, 1994.

SANMARTÍ, N. 10 ideas clave. Evaluar para aprender. : Barcelona: Editorial Graó, 2007

Recebido: 05/03/2019

Aceito: 30/03/2019 